\title{
Autism Spectrum Disorder and miRNA: An Overview of Experimental Models
}

\author{
Giovanni Schepici, Eugenio Cavalli, Placido Bramanti and Emanuela Mazzon * \\ IRCCS Centro Neurolesi “Bonino-Pulejo", Via Provinciale Palermo, Contrada Casazza, 98124 Messina, Italy; \\ giovanni.schepici@irccsme.it (G.S.); eugenio.cavalli@irccsme.it (E.C.); placido.bramanti@irccsme.it (P.B.) \\ * Correspondence: emanuela.mazzon@irccsme.it; Tel.: +39-090-60128172
}

Received: 20 August 2019; Accepted: 2 October 2019; Published: 3 October 2019

\begin{abstract}
Autism spectrum disorder (ASD) is a complex neuropsychiatric disorder characterized by deficits in social interactions, communication, language, and in a limited repertoire of activities and interests. The etiology of ASD is very complex. Genetic, epigenetic, and environmental factors contribute to the onset of ASD. Researchers have shown that microRNAs (miRNAs) could be one of the possible causes associated with ASD. miRNAs are small noncoding mRNAs that regulate gene expression, and they are often linked to biological processes and implicated in neurodevelopment. This review aims to provide an overview of the animal models and the role of the different miRNAs involved in ASD. Therefore, the use of animal models that reproduce the ASD and the identification of miRNAs could be a useful predictive tool to study this disorder.
\end{abstract}

Keywords: autism spectrum disorder; miRNA; experimental models

\section{Introduction}

Autism spectrum disorder (ASD) affects about $2 \%$ of the world population, with a major incidence for males compared to females. Patients with ASD show abnormal, repetitive, and stereotyped behaviors; these abnormalities involve deficits related to cognitive functioning, learning, attention, and sensory development. ASD symptoms coexist with other psychiatric and medical conditions such as intellectual disability, epilepsy, deficit in motor control, attention deficit hyperactivity disorder, gastrointestinal problems, tics, sleep disorders, and anxiety [1]. The meta-analysis of the genes involved in ASD has shown their involvement in numerous biological pathways such as protein synthesis and degradation, dynamics and cytoskeletal structure, synaptic function, and regulation of gene expression. Studies on ASD pathogenesis have shown miRNAs have a direct role in the ASD onset [2,3]. miRNAs are implicated in different cellular processes, such as development, proliferation, differentiation, growth control, homeostasis, and apoptosis. They constitute a class of small noncoding RNAs composed of 20-22 nucleotides. miRNAs are post-transcriptional regulators; they bind to the 3 ' untranslated region (UTR) of messenger RNAs (mRNAs) in order to inhibit the translation or degradation of mRNA. The biogenesis of miRNAs occurs through several steps. In the nucleus, miRNA is transcribed by RNA polymerase II or III into a hairpin structure called primary miRNAs (pri-miRNA). Subsequently, about $25 \%$ of cluster-organized miRNAs are transcribed into a single polycistronic pri-miRNA. Therefore, the pri-miRNA is cleaved from the Drosha and DGCR8 complex in precursor miRNAs (pre-miRNA). The pre-miRNA is translocated by Exportin-5 (XPO5), into the cytoplasm. In the cytoplasm, the pre-miRNAs are split by Dicer RNAase III, creating dissociate duplexes of miRNAs that are potentially mature. One of the two strands associated with Argonaute proteins (AGO 1-4) is called mature miRNA and is composed of 22 nucleotides. Dicer and TAR-RNA binding protein (TRBP) form the RNA-induced silencing complex (RISC) that leads to the target mRNA. Only one filament is incorporated in RISC, while the other is degraded. The RISC-miRNA complex binds the target mRNA and induces or inhibits 
the translation or degradation of mRNA transcription. Since in most cases, imperfect coupling occurs, as a consequence, there is the translational repression of the target protein [4-6]. Reduced levels of miRNAs expressed in the brain, blood, saliva, and other body fluids have been observed in patients with ASD, making them essential biomarkers for ASD identification [7]. This review treats the role of miRNAs in the pathogenesis of ASD. Furthermore, it investigates the animal models of ASD to deepen our knowledge of this type of disorder.

\section{ASD in an Experimental Model}

Several factors contribute to the onset of ASD. Genetic association studies have shown how mutations in some genes can determine the onset of ASD phenotypes, including Fragile X mental retardation 1 (FMR1), methyl-CpG binding protein gene of type 2 (MECP2), Neuroligin (NLGN), Neurexin 1 (NRXN), Tuberous sclerosis 1 and 2 genes (TSC1 and TSC2), Reelin (RELN), Engrailed2 (EN2), and Phosphatase and tensin homolog protein (PTEN). The identification of these genes has led researchers to study animal models that reproduce ASD. Therefore, the use of transgenic mouse models has proven to be a very useful tool to study the pathophysiological mechanisms of ASD and discover new potential therapeutic treatment [8].

The FMR1 Knock-Out (FMR1-KO) is an animal model induced by gene targeting that involves the deletion of exon 5. Researchers have shown that the deletion of the FMR1 gene causes a mental delay in mice as it does in human beings. The FMR1-KO mouse model has shown abnormalities in the development of dendritic spines and pyramidal cells in the cerebral cortex which are involved in neuroplasticity. In addition, this mouse model showed a decrease in social interactions and in the fear of danger as seen in human FXS and ASD [8,9].

Rett syndrome (RTT) is a complex neurological disorder caused by the deletion of $M E C P 2$. This gene is a transcriptional repressor and is located on the $\mathrm{X}$ chromosome. This deletion involves loss of function, determining the RTT. A useful animal model for studying RTT is the MECP2-KO mouse model. Two different MECP2-KO models were generated. One model involves the deletion of exon 3-4, while the other one generated by removal of the entire exon 3 and part of exon 4 and introduction of the nonsense sequence [10,11]. The most severe form of RTT has been observed in MECP2-KO male mice, reporting lethality at 10 weeks of age [12]. In addition, MECP2 Knock-In (MECP2-KI) was generated through the addition of missense mutations in which threonine 158 is converted to alanine [13].

NLGN and NRX are proteins encoded by NLGN and NRX genes. These proteins are involved in cell adhesion, neurotransmission, and synaptic differentiation. Mutations in NLGN and NRX genes may involve behavioral changes and social interactions such as in ASD [14]. On the basis of these mutated genes, some mouse models have been developed [15]. The NLGN1-KO mouse model was induced by the deletion of the first two coding exons. Studies conducted on NLGN1-KO mice have shown deficits in behavioral tests related to anxiety and spatial learning. Furthermore, these animal models have shown an alteration to the stimuli induced by heat and pain in tests. Similarly, these deficits have been observed in patients with ASD [16].

NLGN2 is a gene that codes for the postsynaptic cell adhesion protein NLG2. This protein supports the integrity and functionality of inhibitory synapses and it is also involved in neuropsychiatric and depressive diseases [17]. The NLGN2-KO mouse model involves the deletion of the first coding exon. Studies conducted in the prefrontal cortex and in the hippocampus of NLGN2-KO mice confirmed the role of NLGN2 in maintaining the function of inhibitory synapses [18]. In addition, behavioral studies have shown an increase in anxiety, alterations in the perception of pain-induced stimuli, and a decrease in motor coordination. By contrast, they have shown social behavior interactions and a normal locomotor activity [19].

The NLG3-KO mouse model involves the deletion of exons 2-3; this mouse model has shown the alteration of synaptic plasticity correlated to a mild ASD phenotype. Further, researchers have observed a regular social interaction but an increasing of anxiety in behavior tests [20]. 
NGL3-KI mice were induced by basis replacement of cysteine with arginine in NLGN3 located on chromosome X. Studies conducted on NLG3-KI mice showed a reduction in sociability and an improvement in spatial learning compared to NLG3-KO [21,22]. In addition, Chadman et al. [23], in a study performed on NLG3-KI mice, observed developmental delays and deficits in motor learning.

NRXN1 is a protein present on the presynaptic cell surface and biologically linked to postsynaptic NLGN. In mammals, NRXN1 is encoded by the NRXN1 gene. Further, NRXN1 has two isoforms, Neuroxine $\alpha(N R X \alpha)$ and Neuroxine $\beta(N R X N \beta)$ [24]. Studies on mice have shown that mutations in the NRXN1 gene are associated with ASD, schizophrenia, and other neuropsychiatric disorders. The NRXN1 $\alpha-\mathrm{KO}$ mouse model as a result of the deletion of NRXN-1 $\alpha$ has demonstrated a mild phenotype associated with ASD. Only in females mouse, the researchers have observed hypoactivity. Instead, an increase in aggressive and anxiety-related behaviors has been observed in males. Furthermore, sex-related behavioral alteration in mice is one characteristic of ASD patients; hence, these models are useful for further research on this type of disorder [25].

The SHANK genes encode Src Homology-3 (SH3) and multiple ankyrin repeat domains proteins (SHANKs). Mutations in SHANK includes three genes (SHANK 1-3) related to ASD. The deletion of SHANK3 located on chromosome 22 determines the Phelan-McDermid syndrome, characterized by autistic phenotypes that leads to a linguistic deficit in intellectual and motor development [26]. To better understand the role of these genes in ASD, genetic mutations have been reproduced in mouse models. Studies using SHANK1-KO mice, obtained by the deletion of exons 14 and 15, showed an increase in anxiety, hypoactivity, deficits in motor learning, and conditioning to fear [27]. Researchers employed two types of SHANK2-KO mice, one with deletion of exons 6-7 and the other one with deletion of exon 7. Won et al. [28], using the SHANK2-KO mice model with deletion of exons 6-7, showed in behavioral tests a decrease in interaction and social communication, memory deficits and spatial learning, hyperactivity, and an increase in anxiety-related behavior. Schmeisser et al. [29], reported behavioral alterations in tests such as increased self-cleaning, anxiety, and hyperactivity, exploiting the SHANK2-KO mice model with deletion of the exon 7. Several SHANK3-KO mice have been employed to demonstrate the ASD phenotype. Bozdagi et al. [30], using SHANK3-KO mice with a deletion of exons 4-9, observed normal interest sociability in behavioral tests in mouse puppies. In addition, slight hypoactivity in the open field and in interactions between young male-female couples were observed. Moreover, Yang et al. [31], using SHANK3-KO mice, showed the alteration of motor performance and deficits in recognition of new objects and in learning in tests [30,31]. Wang et al. [32], using SHANK3-KO mice with deletion of exons 4-9, showed alteration of social interaction. Furthermore, deficits in food preferences and motor performance, while normal levels of anxiety have been observed.

Peça et al. [33], using two types of the SHANK3-KO mice model, one with deletion of exons 4-7 and another with a deletion of exons 13-16, have found mostly the ASD phenotype in SHANK3-KO mice with the deletion of exons 13-16. These animals showed a decrease in sociality and male-female interaction. In addition, they showed an excessive increase in anxiety and self-cleaning, with skin lesions often occurring.

Kouser et al. [34], exploiting the SHANK3-KO mice model characterized by the deletion of exon 21 , reported normal social interaction and a complicated nesting in mice. Furthermore, an increase in anxiety and learning and memory deficits in tests was observed.

TSC1 and TSC2 are genes of considerable interest. These genes are located, respectively, on chromosomes 9 and 16, encoding for the proteins Hamartin and Tuberin. Mutations in one of the two TSC genes determine the onset of tuberous sclerosis, an autosomal neurodevelopmental disorder with autistic spectrum symptoms [35]. TSC mutant mouse models have been chosen to deepen the knowledge of this ASD phenotype. The researchers employed two TSC1 mice models, one with deletion of exons 6-8 and the other with deletion of exons 5-7 to generate the nonfunctional copy of this gene. The results of these studies showed how TSC1 mutations in homozygous mice are lethal. By contrast, heterozygous TSC1 mice showed ASD behaviors as a poor male-female interaction within 
the couple and a compromised nest construction [36]. Tsai et al. [37], in a study conducted on the TSC1-KO mice model, observed a significant increase in size and a decrease in the excitability of the Purkinje cells. In behavioral tests, a deficit of social interaction and an increase in repetitive behaviors were shown.

Reith et al. [38], using the TSC2-KO mouse model, observed lethality in homozygous mice, while heterozygote mice showed an ASD-related phenotype. Only females' deficits in social behavior were reported, while the males showed little interest in social novelty and in male-female interaction. Furthermore, deficits in motor performance, learning, and memory were observed.

REEL is a protein involved in neurodevelopment, encoding by RELN, a gene located on chromosome 7. The RELN role is to regulate dendritic budding in GABAergic cerebellar Purkinje cells $[39,40]$. A study performed by Hadj-Sahraoui et al. [41] showed a reduction of Purkinje cells in the cerebellum of RELN heterozygous mutant mice in a period between 3 and 16 months of age. This impairment was observed only in males mice. By contrast, in female mice, no deficits were observed in Purkinje cells. Therefore, these results suggested that RELN exerts its gender-specific action, as demonstrated by the increased incidence of ASD in males [40].

Related to these previous studies is the EN2 gene. This gene is located on chromosome 7 and encoding for the EN2 protein involved in embryonic development of the midbrain and central nervous system. Studies on the EN2-KO mice model showed a decrease in Purkinje cells. Furthermore, researchers demonstrated a reduction in the size of the hippocampus and an ectopic disposition of neurons [42]. Investigations in EN2-KO mice showed behavioral abnormalities such as deficits in social behavior and memory. Therefore, EN2-KO mice showed evidence of morphology and similar behaviors with ASD patients [43].

Another gene of considerable interest is PTEN. This gene is a gene located on chromosome 10 and encodes for the PTEN protein involved in the regulation of the cell cycle. PTEN is important in synaptic plasticity, in neuronal function, and development. PTEN mutations have been associated with ASD phenotypes such as the Cowden, Bannayan-Riley and Proteus syndromes [44]. PTEN-KO mice models are a useful means for planning the study of ASD behaviors. Kwon et al. [45], using the PTEN-KO mice model, showed macrocephaly and also loss of neuronal polarity in the hippocampus and in the cortex. Furthermore, some behavioral anomalies associated with ASD such as anxiety, convulsions, and decreased social interest were experienced in these animals.

It has been observed that women with mutations in the 15q11-q13 gene locus of chromosome 15 determine progeny affected by the Angelman syndrome. Furthermore, it has been observed that the duplication or triplication in locus 15q11-q13 of maternal origin is the most common cytogenetic anomaly associated with ASD. The ubiquitin-protein ligase E3A protein encoded by the UBE3A gene that is located on locus 15q11-q13 was observed. UBE3A plays an important regulatory role in the development of neural circuits and mammalian synaptic plasticity [46]. UBE3A mutations are associated with Angelman syndrome, a disorder characterized by severe somatic and intellectual developmental delay, deficits in speech development, sleep disorders, and motor dysfunction [47]. The transgenic mice model induced by the duplication of $U B E 3 A$ has proven very useful in better understanding this disorder. Behavioral tests conducted on UBE3A mutant mice have shown a reduction in sociability and an increase in self-care [48]. Table 1 summarizes the list of knockout mouse models related to autism spectrum disorders. 
Table 1. Genes associated with autism spectrum disorder (ASD) for which a Knock-Out model is provided. The column variants are "AutDB variants", including the variants in the protein-coding region that is AutDB-related and with a familial origin.

\begin{tabular}{|c|c|c|c|c|}
\hline Human Gene & Locus & Aut DB Variants & $\begin{array}{l}\text { Associated } \\
\text { Disease }\end{array}$ & Model \\
\hline \multirow[t]{7}{*}{ FMR1 } & \multirow[t]{7}{*}{ Xq27.3 } & p.Lys547Glu & \multirow[t]{7}{*}{ Fragile $X$ syndrome } & \multirow[t]{7}{*}{ FMR1-KO } \\
\hline & & p.Glu360Gly & & \\
\hline & & p.Ser27Ter & & \\
\hline & & p.Arg138Gln & & \\
\hline & & p.Ile580fsTer9 & & \\
\hline & & p.Gly266Glu & & \\
\hline & & p.Met140IlefsTer3 & & \\
\hline \multirow[t]{33}{*}{ МECP2 } & \multirow[t]{33}{*}{ Xq28 } & p.Glu406Ter & \multirow[t]{33}{*}{ Rett syndrome } & \multirow[t]{33}{*}{ MECP2-KO } \\
\hline & & p.Ala140Val & & \\
\hline & & p.Arg167Trp & & \\
\hline & & p.Pro388_Pro467del & & \\
\hline & & p.Pro322Ser & & \\
\hline & & p.Ala214Gly & & \\
\hline & & p.Thr240Ser & & \\
\hline & & p.Ala370Thr & & \\
\hline & & p.Pro199Arg & & \\
\hline & & p.Glu483Ter & & \\
\hline & & p.Glu318Asp & & \\
\hline & & p.Val320His & & \\
\hline & & p.Arg354_Val412delins41 & & \\
\hline & & p.Glu495Ter & & \\
\hline & & p.Gly185Val & & \\
\hline & & p.Arg167Trp & & \\
\hline & & p.Arg309Trp & & \\
\hline & & p.Ala140Val & & \\
\hline & & p.Pro398fsTer & & \\
\hline & & p.Glu483Ter & & \\
\hline & & p.Glu495Ter & & \\
\hline & & p.Arg190His & & \\
\hline & & p.Arg133Cys & & \\
\hline & & p.Arg306Cys & & \\
\hline & & p.Arg133Cys & & \\
\hline & & p.Pro389Ter & & \\
\hline & & p.Arg $470 \mathrm{His}$ & & \\
\hline & & p.Asp147Glu & & \\
\hline & & p.Pro376Ser & & \\
\hline & & p.Gly249fs & & \\
\hline & & p.Val380CysfsTer27 & & \\
\hline & & p.Pro403Arg & & \\
\hline & & p.Glu406Lys & & \\
\hline
\end{tabular}


Table 1. Cont.

\begin{tabular}{|c|c|c|c|c|}
\hline Human Gene & Locus & Aut DB Variants & $\begin{array}{c}\text { Associated } \\
\text { Disease }\end{array}$ & Model \\
\hline \multirow[t]{8}{*}{ NLGN3 } & \multirow[t]{8}{*}{ Xq13.1 } & p.Arg451Cys & & \multirow[t]{8}{*}{ NLGN3-KO } \\
\hline & & p.Thr632Ala & & \\
\hline & & p.Val321Ala & & \\
\hline & & p.Val306Met & & \\
\hline & & p.Trp463Ter & & \\
\hline & & p.Arg617Trp & & \\
\hline & & p.Thr429fs & & \\
\hline & & p.Thr449fs & & \\
\hline \multirow[t]{11}{*}{ NLGN4X } & \multirow[t]{11}{*}{ Xp22.32-p22.31 } & p.Lys378Arg & & \\
\hline & & p.Gln329Ter & & \\
\hline & & p.Arg766Gln & & \\
\hline & & p.Gly84Arg & & \\
\hline & & p.Ala283Thr & & \\
\hline & & p.Gly99Ser & & \\
\hline & & p.Lys378Arg & & \\
\hline & & p.Val403Met & & \\
\hline & & p.Arg704Cys & & \\
\hline & & p.Glu418AspfsTer12 & & \\
\hline & & p.Gln274Ter & & \\
\hline \multirow[t]{10}{*}{ TSC1 } & \multirow[t]{10}{*}{$9 q 34.13$} & p.His732Tyr & \multirow[t]{10}{*}{ Tuberous sclerosis } & \\
\hline & & p.Lys587Arg & & \\
\hline & & p.Ser403Leu & & \\
\hline & & p.Thr360Asn & & \\
\hline & & p.Arg336Trp & & \\
\hline & & p.Thr360Asn & & \\
\hline & & p.Pro448Ser & & \\
\hline & & p.Ala186Thr & & \\
\hline & & p.Asn6Asp & & \\
\hline & & p.Glu190fs & & \\
\hline \multirow[t]{12}{*}{ TSC2 } & \multirow[t]{12}{*}{$16 \mathrm{p} 13.3$} & p.Lys533Gln & \multirow[t]{12}{*}{ Tuberous sclerosis } & \\
\hline & & p.Ala678Thr & & \\
\hline & & p.Glu984Gln & & \\
\hline & & p.Ala1097Val & & \\
\hline & & p.Ser1698Arg & & \\
\hline & & p.Ile64Val & & \\
\hline & & p.His152Asp & & \\
\hline & & p.Lys533Gln & & \\
\hline & & p.Lys954Arg & & \\
\hline & & p.Glu984Gln & & \\
\hline & & p.Ala1429Ser & & \\
\hline & & p.Glu234fs & & \\
\hline
\end{tabular}


Table 1. Cont.

\begin{tabular}{|c|c|c|c|c|}
\hline Human Gene & Locus & Aut DB Variants & $\begin{array}{c}\text { Associated } \\
\text { Disease }\end{array}$ & Model \\
\hline \multirow[t]{5}{*}{ TSC2 } & \multirow[t]{5}{*}{$16 \mathrm{p} 13.3$} & p.Val1034Ile & \multirow[t]{5}{*}{ Tuberous sclerosis } & \\
\hline & & p.Asp647Asn & & \\
\hline & & p.Val296Met & & \\
\hline & & p.Leu361Val & & \\
\hline & & p.Arg622Trp & & \\
\hline \multirow[t]{36}{*}{$R E L N$} & \multirow[t]{36}{*}{$7 q 22.1$} & p.Gly370Arg & & \\
\hline & & p.Asn1159Lys & & \\
\hline & & p.Ser1719Leu & & \\
\hline & & p.Arg1742Gln & & \\
\hline & & p.Val1762Ile & & \\
\hline & & p.Arg2290His & & \\
\hline & & p.Thr2718Ala & & \\
\hline & & p.Gly1280Glu & & \\
\hline & & p.Arg255Trp & & \\
\hline & & p.Arg2292Cys & & \\
\hline & & p.Thr1904Met & & \\
\hline & & p.Arg1727Trp & & \\
\hline & & p.Pro1580Leu & & \\
\hline & & p.Pro3379Arg & & \\
\hline & & p.Ile3374Val & & \\
\hline & & p.Pro2245Arg & & \\
\hline & & p.Leu2057Val & & \\
\hline & & p.Arg822Gly & & \\
\hline & & p.Arg2242Ser & & \\
\hline & & p.Leu522Phe & & \\
\hline & & p.Arg3439Gln & & \\
\hline & & p.Val3426Ile & & \\
\hline & & p.Asn2535Lys & & \\
\hline & & p.Val2372Met & & \\
\hline & & p.Asp2309Asn & & \\
\hline & & p.Asp2309Asn & & \\
\hline & & p.Arg1742Gln & & \\
\hline & & p.Glu1410Lys & & \\
\hline & & p.Leu411Ile & & \\
\hline & & p.Val3426Ile & & \\
\hline & & p.Lys751Thr & & \\
\hline & & p.Tyr1183Cys & & \\
\hline & & p.Pro638Leu & & \\
\hline & & p.Ala3327Ser & & \\
\hline & & p.Pro638Leu & & \\
\hline & & p.Arg1727Gln & & \\
\hline
\end{tabular}


Table 1. Cont.

\begin{tabular}{|c|c|c|c|c|}
\hline Human Gene & Locus & Aut DB Variants & $\begin{array}{c}\text { Associated } \\
\text { Disease }\end{array}$ & Model \\
\hline \multirow[t]{17}{*}{ multirow1* } & \multirow[t]{17}{*}{ RELN 7q22.1 } & p.Ser142Asn & & \\
\hline & & p.Ala3281GlnfsTer11 & & \\
\hline & & p.Gly1113Glu & & \\
\hline & & p.Tyr2154Cys & & \\
\hline & & p.Gly2802Arg & & \\
\hline & & p.Gly2783Cys & & \\
\hline & & p.His798Asn & & \\
\hline & & p.Pro844Leu & & \\
\hline & & p.Asp763Gly & & \\
\hline & & p.Pro672Leu & & \\
\hline & & p.Tyr723Cys & & \\
\hline & & p.Ser2513Cys & & \\
\hline & & p.Ala2545Val & & \\
\hline & & p.Trp1083fs & & \\
\hline & & p.Pro1340Leu & & \\
\hline & & p.Asp2309Asn & & \\
\hline & & p.Gln3313Arg & & \\
\hline EN2 & $7 q 36.3$ & p.Pro142Arg & & EN2-KO \\
\hline \multirow[t]{18}{*}{ PTEN } & \multirow[t]{18}{*}{$10 q 23.31$} & p.Tyr178Ter & & \\
\hline & & p.Asp22Glu & & \\
\hline & & p.Gln214Ter & & \\
\hline & & p.Met134Ile & & \\
\hline & & p.Arg130Ter & & \\
\hline & & p.Leu139Ter & & \\
\hline & & p.Arg173His & & \\
\hline & & p.Trp274Leu & & \\
\hline & & p.Met134Thr & & \\
\hline & & P.Leu182Ser & & \\
\hline & & p.Ile50Thr & & \\
\hline & & p.Val133Ile & & \\
\hline & & p.Lys164Asn & & \\
\hline & & p.Ser59Ter & & \\
\hline & & p.Arg233Ter & & \\
\hline & & p.Ser170Arg & & \\
\hline & & p.Gly136fs & & \\
\hline & & p.Arg154fs & & \\
\hline \multirow[t]{6}{*}{ UBE3A } & \multirow[t]{6}{*}{$15 q 11.2$} & p.Ala201Thr & \multirow[t]{6}{*}{ Angelman } & \\
\hline & & p.Ala178Thr & & \\
\hline & & p.Ala198Thr & & \\
\hline & & p.192_193del & & \\
\hline & & p.Arg506Cys & & \\
\hline & & p.Ala599fs & & \\
\hline
\end{tabular}


Researchers also used nongenetic animal models to study ASD behaviors. A mouse model that was used is that induced by compounds that determine maternal immune activation (MIA) during gestation. C57BL/6 mice were exposed to polyinosinic:polycytidylic acid (Poly I:C) during pregnancy to mimic viral and bacterial infection. The progeny resulting from (Poly I:C) exposure showed different immunological, neurological, and behavioral deficits such as in ASD. Researchers found little sociability in the behavioral tests [49]. Another animal model of ASD used prenatal exposure to valproic acid (VPA), an antiepileptic drug that stabilizes mood. This model is a useful tool for investigating the etiology and biology of ASD [50]. The researchers showed how, in rodents, prenatal exposure to VPA could induce behavioral deficits such as those seen in ASD. Therefore, based on these observations, in 1996, the first model was created by administering a single dose of $350 \mathrm{mg} / \mathrm{kg}$ of VPA to rat embryos [51]. ASD can be defined only by behavioral observations. Male rats showed numerous behaviors associated with ASD. The rats showed an increase in repetitive and compulsive behaviors and a reduction in sociality, attention, and spinal sensitivity induced by pain. In addition, the researchers observed deficits in olfactory discrimination, reduction in body weight, and delays in motor development [52]. Behavioral tests evaluating interaction and social communication were performed. Furthermore, the labyrinth test was used to observe repetitive behaviors. Anxiety levels were assessed by measuring the time spent with open arms in the labyrinth. Further tests regarding food preferences showed how VPA rats tend to eat less tasty foods compared to the control rat [53]. Based on these observations, the VPA rodent model can be classified as useful for the study of neurobiology and the behavior of ASD [54].

\section{3. miRNA and ASD}

Underlying the pathogenesis of ASD, recent studies have shown that miRNAs are involved in the development of the examined disorder. The mouse models, designed after the discovery of the mutated gene responsible for the ASD form, proved to be useful tools for researchers to identify the causes of the disease.

Edbauer et al., using FMR1-KO mice, demonstrated that the silencing of the FMR1 gene encoding for the Fragile X mental retardation protein (FMRP) determines the onset of FXS. The researchers observed that miRNA-125b and miRNA-132 are important in the regulation of FMRP translation. In addition, these miRNAs act differently on the morphology of the spine and synaptic plasticity in the hippocampal neurons. The identification of miRNAs in the brain of these mice was performed by extracting the FMRP protein and by marking it with specific antibodies to identify the mRNA targets of the protein. The subsequent quantitative analysis allowed identifying 12 miRNAs with FMRP as a target. Moreover, the overexpression of miRNA-125b and miRNA-132 in hippocampal neurons after plasmid transfection was evaluated. miRNA-125b upregulation caused the development of long and thin spines, while miRNA-132 upregulation led to an increase in the average width of the spines and reduced spinal density. miRNA-125b upregulation led to a reduction in synaptic transmission, as demonstrated by long and thin spines. By contrast, miRNA-132 upregulation led to an increase in synaptic frequency, as confirmed by the width of the spines [55]. In addition, it was also demonstrated that miRNA-125b targets the NR2A subunit of the N-methyl-D-aspartate receptor (NDMAR) that influences synaptic plasticity and memory consolidation. Therefore, the study of the NDMAR function in these mice could lead to new strategies for the treatment of FXS.

Zongaro et al., using FMR1-KI mice with tremor/ataxia syndromes associated with Fragile $\mathrm{X}$ (FXTAS), observed behaviors associated with ASD. FXTAS is a more severe form of FXS, in which the overexpression of FMR1 that occurs in adulthood causes developmental and behavioral deficits. In tests of the aquatic labyrinth, these mice aged between 52 and 72 weeks showed numerous cognitive deficits. The researchers, using luciferase activity, which binds the 3'UTR sequence of the FMR1 gene, showed how miRNA-101, miRNA129-5p, and miRNA-221 regulate the expression of FMR1 in the brain. In addition, the synaptic expression levels of miRNA-129-5p, miRNA-221, and miRNA-101 were compared with miRNA-134 levels. The expression of miRNA-221 was not detectable during embryonic life. While the authors reported that miRNA-129-5p expression was increased in the postnatal period, 
miRNA-221 was increased in the adult phase. In addition, the study of Zongaro et al. reported that miRNA-221 upregulation determines loss MECP2 function such as in RTT [56].

The results on KI mice showed that overexpression of FMR1 causes synaptopathy, a characteristic of phenotypes of ASD, such as in FXSTAS. In addition, in the study of Zongaro et al., it was reported that miRNA-221 upregulation determines the loss of MECP2 function such as in RTT.

Urdinguio et al., using MECP2-KO mice, observed the dysregulation of some miRNAs. The subsequent brain transcriptomic analysis showed that miRNA-146a, miRNA-146b, miRNA-130, miRNA-122a, miRNA-342, and miRNA-409 were downregulated, while miRNA-29b, miRNA-329, miRNA-199b, miRNA-382, miRNA-296, miRNA-221, and miRNA-92 were upregulated. Urdinguio et al. demonstrated that miRNA-146a and miRNA-146b are involved in ASD etiopathogenesis. miRNA-146a and miRNA-146b targeted the 3'-UTR sequence of interleukin-1 receptor-associated kinase 1 (IRAK1) encoded by the IRAK1 gene. The IRAK1 protein, engaging with one of the two serine/threonine kinases, binds the interleukin-1 triggering inflammatory function [57]. The researchers subsequently evaluated the regulatory role of miRNA-146a and miRNA-146b in a neuronal context, transfecting them into murine neuroblastoma cells [58]. They showed that in healthy brain conditions, miRNA-146a and miRNA-146b determine the down-expression of IRAK1. In addition, the downregulation of miRNA-146a and miRNA-146b causes IRAK1 overexpression by inducing an inflammatory state of brain tissue and by contributing to the phenotype RTT [59].

Zhang et al., using MECP2 transgenic mice, showed how this gene is implicated in the pain pathway by acting as an analgesic mediator. The study proved deficits in social interaction, anxiety, and motor functions. The researchers observed that MECP2 overexpression is linked via the $\mathrm{p}-\mathrm{CREB} / \mathrm{miR}-132$ protein pathway. This complex, binding to the cAMP, generates the reduction of pain induced in these mice. miRNA-132 targets the $3^{\prime} \mathrm{UTR}$ sequence of $M E C P 2$ when it is upregulated and inhibits the level expression at the post-transcriptional level of $M E C P 2$. The downregulation of miRNA-132 leads to an increase in $M E C P 2$ expression related to increased analgesic response to mechanical and thermal stimulation [60].

Lyu et al., showed in an in vitro study using primary mouse neurons, that miRNA-137 and miRNA-132 regulate MECP2 and PTEN expression. The MECP2 knockout upregulated miRNA-137, which in turn leads to PTEN down-expression. Furthermore, PTEN knockout was observed to upregulate miRNA-132. Therefore, the study showed how the genes MECP2 and PTEN regulate their expression through miRNAs [61].

Cheng et al. characterized a new KO mouse model for MIR137. They observed how this gene is essential for mouse postnatal development, which is important as the loss of function of MIR137 has been observed in ASD. Furthermore, they demonstrated that the partial loss of miRNA-137 function causes alterations in synaptic plasticity and behavior [62].

Cortabitarte et al. performed in silico and in vitro studies on the SHANK genes, which code for postsynaptic scaffold proteins. In the in silico study, they identified a single miRNA-137 binding site in the SHANK2 gene. In the in vitro study, on mouse hippocampal neurons, they showed through Western blot analysis how the upregulation of miRNA-137 reduces the SHANK2 expression levels. Therefore, the overexpression of miRNA-137 involves the downregulation of the SHANK2, an important protein involved in the development of the postsynaptic structure [63].

$U B E 3 A$ plays an important role in neural development and synaptic plasticity. Vatsa et al., using the Angelman syndrome mouse model, showed how miRNA-708 has been downregulated. In addition, they observed that miRNA-708 targets the NNAT gene that encodes for Neuronatin protein (NNAT). This gene is involved in the regulation of ion channels during brain development. The researchers observed that downregulation of miRNA-708 leads to an increase in intracellular Ca2 ${ }^{+}$. Moreover, they observed the phosphorylation of Calcium/calmodulin-dependent protein kinase type II alpha chain $(C A M K I I \alpha)$, encoded by the CAMK2A gene. CAMKII $\alpha$ protein is involved in synaptic plasticity. In addition, an increase was observed in NNAT levels and GABAergic neurons. The results of the study showed how miRNA-708 acts on NNAT mediating the aberrant $\mathrm{Ca}_{2}{ }^{+}$signaling, thus contributing to the pathogenesis of the Angelman syndrome [64]. 
Valluy et al., using the $U B E 3 A-K O$ mouse model, demonstrated that $U B E 3 A 1$ transcription encodes an inactive UBE3A1 protein. The UBE3A1 protein, as it lacks the E3 ligase responsible for catalytic activity, prevents dendritic growth and promotes the maturation of the dendritic spine in these mice. The study showed how the miR379-410 cluster in particular miRNA-134 competes with UBE3A1 through the 3'-UTR sequence for the mRNA target. During neural development, it was observed that both the down-expression of $U B E 3 A 1$ and the upregulation of the miR379-410 cluster containing miRNA-134 favored the dendritic growth but not that of spine maturation. Therefore, the dysregulation of the miR379-410 cluster and miRNA-134 can determine the onset of deficits in neurodevelopment as in ASD [46].

Lackinger et al., using the miR379-410-KO mouse model, observed the correlation between loss of miR379-410 functions and alteration in social behavior. MiR379-410 is a cluster located on chromosome 12 in mice. The $\mathrm{KO}$ of the miR379-410-KO mouse model showed an increase in excitatory synaptic transmission in the hippocampus, resulting in altered social behavior and increased anxiety. Furthermore, no memory deficits were observed in social and object recognition tests. In conclusion, this study showed that the miR379-410 cluster influences the development of social behaviors in mice as in patients with ASD [65].

Hirsch et al. evaluated the effects of treatment with resveratrol (RSV), an antioxidant and anti-inflammatory molecule, in a rat model of ASD induced by the administration of VPA. The study showed that RSV prevents behavioral deficits in VPA rats. Furthermore, the expression of circulating miRNAs in VPA rats was estimated before and after the treatment with RSV. An increase in the miRNA-134-5p level was identified, while treatment with RSV prevented the alteration of these miRNA levels. Furthermore, the expression of miRNAs of patients with ASDs compared to healthy patients was evaluated. The results showed an increase in the levels of miRNA134-5p and miRNA138-5p in patients with ASD [66].

miRNA-134-5p and miRNA-138-5p acted in the brain, dynamically regulating the cytoskeleton through two antagonistic pathways. miRNA-134-5p could negatively affect the size of dendritic spines in postsynaptic sites and inhibit the translation of the protein domain kinase 1 (LIMK1) encoded by the LIMK1 gene. By contrast, miRNA-138-5p is involved in the development of dendritic spines. miRNA-138-5p regulates the expression of the acyl thioesterase 1 (APT1) protein encoded by the APT1 gene. APT1 is an enzyme that regulates the depalmitoylation of the $\mathrm{G} \alpha$ protein and, consequently, the morphogenesis of the dendritic spine. The results of the study demonstrate an important mechanism of control on vertebral column morphogenesis exerted by miRNA-138-5p on APT1 expression [67].

Dai et al., using the VPA-rat model, shown that miRNA-34a regulates the expression of BCL2, although the mechanisms are not entirely known. They observed in the cerebral cortex of VPA rats, immediately after birth, the upregulation of miRNA-34a and BCL2 down-expression. Consequently, the miRNA-34a/Bcl-2 signaling pathway could be an important way to activate ASD in VPA rats [68]. Moreover, the exposure to VPA in rats caused alterations in the miRNA-181c and miRNA-30d profiles. Furthermore, these animals produced an enlarged amygdala, thus confirming the behavioral alterations observed also in ASD patients. The amygdala transcriptomic analysis showed alteration of the neuronal morphology that can be traced to a post-transcriptional process regulated by miRNAs. The results of these analyses suggested that high levels of miRNA-181c and miRNA-30d can provide a dysregulated expression on their mRNA targets that encode proteins involved in neuronal development and that contribute to the pathophysiology of ASD $[69,70]$.

Schneider et al., in a study on the amygdala of VPA rats, evaluated miRNA expression and studied the behaviors. Subsequent analyses showed the upregulation of miRNA-181c and miRNA-30d in VPA rats. Further, analysis of the genes containing a binding site for miRNA-30 and miRNA-181c was performed. miRNA-30d targets numerous genes whose function is mainly associated with the development of the nervous system. The researchers also observed motor deficits and an increase in anxiety in behavioral tests. In conclusion, the dysregulation of these miRNAs can be considered an important event for neurodevelopment, determining the onset of characteristics similar to ASD as 
evidenced in the VPA rat model [71]. Table 2 summarizes all miRNA correlated to autism spectrum disorders with their functions and relative mice model.

Table 2. MiRNAs dysregulated in ASD and identified in animal models.

\begin{tabular}{|c|c|c|c|}
\hline miRNA & Function & Reference & Models \\
\hline miRNA-125b & $\begin{array}{l}\text { miRNA-125b regulates the expression of FMR1; it is } \\
\text { upregulated with the FMR1 Knock-out. It contributes to } \\
\text { the alteration of synaptic plasticity as in FXS. }\end{array}$ & [55] & KO mice \\
\hline miRNA-132 & $\begin{array}{l}\text { miRNA- } 132 \text { and miRNA- } 125 \mathrm{~b} \text { are involved in synaptic } \\
\text { plasticity. Furthermore, miRNA- } 132 \text { targets the } 3 \text { 'UTR } \\
\text { sequence of } M E C P 2 \text { and prevents its expression. As a } \\
\text { result of the pain, it is downregulated, inducing an } \\
\text { increase in the analgesic response and in the expression of } \\
M E C P 2 \text {. Furthermore, it is involved through the } \\
\text { p-CREB/miR-132 pathway in the regulation of expression } \\
\text { between } P T E N \text { and } M E C P 2 \text {. }\end{array}$ & {$[55,60,61]$} & KO mice \\
\hline $\begin{array}{l}\text { miRNA-146a, } \\
\text { miRNA-146b }\end{array}$ & $\begin{array}{l}\text { miRNA-146a and miRNA-146b were observed in the RTT } \\
\text { syndrome. A study on the MECP2-KO mice model } \\
\text { observed that they are downregulated by inducing } \\
\text { overexpression of the IRAK1 gene which leads to an } \\
\text { inflammatory state of the brain tissue such as in ASD. }\end{array}$ & [57-59] & $\mathrm{KO}$ mice \\
\hline miRNA-137 & $\begin{array}{l}\text { When miRNA-137 is downregulated, it influences the } \\
\text { expression of many genes implicated in } \\
\text { neurodevelopment. This means they lead to learning and } \\
\text { memory deficits, and they are therefore associated with } \\
\text { ASD. Furthermore, miRNA-137 is associated with } \\
\text { SHANK2, as both are expressed in synapses. miRNA-137 } \\
\text { targets the 3'UTR sequence of SHANK2 so as to repress the } \\
\text { expression of the SHANK2 protein. }\end{array}$ & [61-63] & KO mice \\
\hline miRNA-134 & $\begin{array}{c}\text { The KO of } U B E 3 A \text { allowed observing an upregulation of } \\
\text { miRNA-134 by belonging to the miRNA379-410 cluster } \\
\text { that regulates synaptic plasticity. Furthermore, the } \\
\text { UBE3A1 RNA transcript acts as a rival endogenous } \\
\text { dendritic RNA for miRNA379-410. }\end{array}$ & [65] & $\mathrm{KO}$ mice \\
\hline miRNA-134-5p & $\begin{array}{l}\text { miRNA-134-5p is overexpressed in the brains of patients } \\
\text { with ASD; it controls the development of the spine. }\end{array}$ & {$[66,67]$} & VPA rats \\
\hline miRNA-138-5p & $\begin{array}{l}\text { miRNA138-5p is implicated in the development changes } \\
\text { of dendritic spines and in the narrowing of the spine. }\end{array}$ & {$[66,67]$} & VPA rats \\
\hline miRNA-34a & $\begin{array}{l}\text { VPA regulates miRNA-34a levels which in turn regulate } \\
B C L 2 \text { expression. In the cerebral cortex of VPA rats } \\
\text { immediately after birth, the upregulation of miRNA-34a } \\
\text { and a reduction in } B C L 2 \text { expression were observed. }\end{array}$ & [68] & VPA rats \\
\hline miRNA-181c & $\begin{array}{l}\text { miRNA-181c is upregulated in the amygdala of ASD } \\
\text { patients; its function is associated with the development of } \\
\text { the nervous system. }\end{array}$ & [69-71] & VPA rats \\
\hline miRNA-30d & $\begin{array}{l}\text { miRNA-30d is also upregulated in VPA rats, and its } \\
\text { function is associated with the development of the } \\
\text { nervous system. }\end{array}$ & [69-71] & VPA rats \\
\hline
\end{tabular}

\section{Conclusions}

Research has revealed that ASD has a genetic basis, as well as epigenetic and environmental factors may contribute to its development. Based on these observations, researchers planned animal models capable of reproducing and studying the ASD phenotype. Animal models are an important means of linking specific changes in miRNA biogenesis and the molecular role they play in the etiopathogenesis 
of neuropathological and behavioral abnormalities. Murine models have been reproduced based on mutations found in patients with ASD. Additionally, nongenetic ASD animal models obtained from prenatal exposure to VPA or induced by immune activation following infection have been useful for researchers to study this disorder. In recent years, miRNAs have attracted the attention of researchers by showing promising results and by signifying that these molecules have a straight role in the pathogenesis of ASD. Research showed that several miRNAs, including miRNA-125b, miRNA-132, miRNA-146a, miRNA-146b, miRNA-137, miRNA-134, miRNA-134-5p, miRNA-138-5p, miRNA-34a, miRNA-181c, and miRNA-30d, are directly linked to ASD. Therefore, since miRNAs regulate the expression of several genes involved in neurodevelopment, they are directly involved in ASD. Furthermore, on the basis of these observations, researchers have identified miRNAs as an important tool to identify new therapies in order to obtain the best and supportive results for ASD. Moreover, miRNAs can be identified as potential biomarkers and validated for the diagnosis and prognosis of the autistic spectrum or can even be used to distinguish different phenotypes of a specific psychiatric disorder, such as ASD.

Author Contributions: Conceptualization, E.M. and P.B.; writing-original draft preparation, G.S. and E.C.; writing - review and editing, E.M. and P.B.

Funding: This study was supported by current research funds 2019 of IRCCS "Centro Neurolesi Bonino Pulejo".

Acknowledgments: We would like to thank the Ministry of Health (Italy) for having provided the research funds 2019 to support the study.

Conflicts of Interest: The authors declare there is no conflict of interest.

\section{References}

1. Faras, H.; Al Ateeqi, N.; Tidmarsh, L. Autism Spectrum Disorders. Ann. Saudi. Med. 2010, 30, 295-300. [CrossRef] [PubMed]

2. Meek, S.E.; Lemery-Chalfant, K.; Jahromi, L.B.; Valiente, C. A review of gene-environment correlations and their implications for autism: A conceptual model. Psychol. Rev. 2013, 120, 497-521. [CrossRef] [PubMed]

3. Miyake, K.; Hirasawa, T.; Koide, T.; Kubota, T. Epigenetics in Autism and Other Neurodevelopmental Diseases. Genome Ed. 2012, 724, 91-98.

4. Winter, J.; Jung, S.; Keller, S.; Gregory, R.I.; Diederichs, S. Many roads to maturity: microRNA biogenesis pathways and their regulation. Nature 2009, 11, 228-234. [CrossRef]

5. Abdelfattah, A.M.; Park, C.; Choi, M.Y. Update on Non-Canonical Micrornas. Biomol. Concepts 2014, 5, 275-287. [CrossRef]

6. Hammond, S.M. An Overview of Micrornas. Adv. Drug Deliv. Rev. 2015, 87, 3-14. [CrossRef] [PubMed]

7. Hicks, S.D.; Middleton, F.A. A Comparative Review of microRNA Expression Patterns in Autism Spectrum Disorder. Front. Psychol. 2016, 7, 176. [CrossRef]

8. Bey, A.L.; Jiang, Y.-H. Overview of Mouse Models of Autism Spectrum Disorders. Curr. Protoc. Pharmacol. 2014, 66, 5-66.

9. Chonchaiya, W.; Au, J.; Schneider, A.; Hessl, D.; Harris, S.W.; Laird, M.; Mu, Y.; Tassone, F.; Nguyen, D.V.; Hagerman, R.J. Increased Prevalence of Seizures in Boys Who Were Probands with the Fmr1 Premutation and Co-Morbid Autism Spectrum Disorder. Hum. Genet. 2012, 131, 581-589. [CrossRef]

10. Guy, J.; Hendrich, B.; Holmes, M.; Martin, J.E.; Bird, A. A mouse Mecp2-null mutation causes neurological symptoms that mimic Rett syndrome. Nat. Genet. 2001, 27, 322-326. [CrossRef]

11. Calfa, G.; Percy, A.K.; Pozzo-Miller, L. Experimental models of Rett syndrome based on Mecp2 dysfunction. Exp. Boil. Med. 2011, 236, 3-19. [CrossRef] [PubMed]

12. Chen, R.Z.; Akbarian, S.; Tudor, M.; Jaenisch, R. Deficiency of Methyl-Cpg Binding Protein-2 in Cns Neurons Results in a Rett-Like Phenotype in Mice. Nat. Genet. 2001, 27, 231-327. [CrossRef] [PubMed]

13. Goffin, D.; Allen, M.; Zhang, L.; Amorim, M.; Wang, I.T.; Reyes, A.R.; Mercado-Berton, A.; Ong, C.; Cohen, S.; $\mathrm{Hu}$, L.; et al. Rett Syndrome Mutation Mecp2 T158a Disrupts DNA Binding, Protein Stability and Erp Responses. Nat. Neurosci. 2011, 15, 274-283. [CrossRef] 
14. Zatkova, M.; Bakoš, J.; Hodosy, J.; Ostatníková, D. Synapse alterations in autism: Review of animal model findings. Biomed. Pap. 2016, 160, 201-210. [CrossRef] [PubMed]

15. McGee, A.; Li, G.; Lu, Z.; Qiu, S. Convergent synaptic and circuit substrates underlying autism genetic risks. Front. Boil. 2014, 9, 137-150. [CrossRef] [PubMed]

16. Blundell, J.; Blaiss, C.A.; Etherton, M.R.; Espinosa, F.; Tabuchi, K.; Walz, C.; Bolliger, M.F.; Südhof, T.C.; Powell, C.M. Neuroligin-1 deletion results in impaired spatial memory and increased repetitive behavior. J. Neurosci. 2010, 30, 2115-2129. [CrossRef] [PubMed]

17. Heshmati, M.; Aleyasin, H.; Menard, C.; Christoffel, D.J.; Flanigan, M.E.; Pfau, M.L.; Hodes, G.E.; Lepack, A.E.; Bicks, L.K.; Takahashi, A.; et al. Cell-type-specific role for nucleus accumbens neuroligin-2 in depression and stress susceptibility. Proc. Natl. Acad. Sci. USA 2018, 115, 1111-1116. [CrossRef] [PubMed]

18. Liang, J.; Xu, W.; Hsu, Y.-T.; Yee, A.X.; Chen, L.; Südhof, T.C. Conditional neuroligin-2 knockout in adult medial prefrontal cortex links chronic changes in synaptic inhibition to cognitive impairments. Mol. Psychiatry 2015, 20, 850-859. [CrossRef]

19. Blundell, J.; Tabuchi, K.; Bolliger, M.F.; Blaiss, C.A.; Brose, N.; Liu, X.; Sudhof, T.C.; Powell, C.M. Increased Anxiety-Like Behavior in Mice Lacking the Inhibitory Synapse Cell Adhesion Molecule Neuroligin 2. Genes Brain Behav. 2009, 8, 114-126. [CrossRef] [PubMed]

20. Radyushkin, K.; Hammerschmidt, K.; Boretius, S.; Varoqueaux, F.; Ronnenberg, A.; Winter, D.; Frahm, J.; Fischer, J.; Brose, N.; Ehrenreich, H.; et al. Neuroligin-3-deficient mice: model of a monogenic heritable form of autism with an olfactory deficit. Genes Brain Behav. 2009, 8, 416-425. [CrossRef]

21. Jaramillo, T.C.; Liu, S.; Pettersen, A.; Birnbaum, S.G.; Powell, C.M. Autism-related neuroligin-3 mutation alters social behavior and spatial learning. Autism Res. 2014, 7, 264-272. [CrossRef] [PubMed]

22. Tabuchi, K.; Blundell, J.; Etherton, M.R.; Hammer, R.E.; Liu, X.; Powell, C.M.; Südhof, T.C. A neuroligin-3 mutation implicated in autism increases inhibitory synaptic transmission in mice. Science 2007, 318, 71-76. [CrossRef] [PubMed]

23. Chadman, K.K.; Gong, S.; Scattoni, M.L.; Boltuck, S.E.; Gandhy, S.U.; Heintz, N.; Crawley, J.N. Minimal aberrant behavioral phenotypes of neuroligin-3 R451C knockin mice. Autism Res. 2008, 1, 147-158. [CrossRef] [PubMed]

24. Reissner, C.; Runkel, F.; Missler, M. Neurexins. Genome. Biol. 2013, 14, 213. [CrossRef] [PubMed]

25. Grayton, H.M.; Missler, M.; Collier, D.A.; Fernandes, C. Altered Social Behaviours in Neurexin 1alpha Knockout Mice Resemble Core Symptoms in Neurodevelopmental Disorders. PLoS ONE 2013, 8, e67114. [CrossRef] [PubMed]

26. Jiang, Y.-H.; Ehlers, M.D. Modeling autism by SHANK gene mutations in mice. Neuron 2013, 78, 8-27. [CrossRef] [PubMed]

27. Wöhr, M.; Roullet, F.I.; Hung, A.Y.; Sheng, M.; Crawley, J.N. Communication Impairments in Mice Lacking Shank1: Reduced Levels of Ultrasonic Vocalizations and Scent Marking Behavior. PLOS ONE 2011, 6, e20631. [CrossRef]

28. Won, H.; Lee, H.-R.; Gee, H.Y.; Mah, W.; Kim, J.-I.; Lee, J.; Ha, S.; Chung, C.; Jung, E.S.; Cho, Y.S.; et al. Autistic-like social behaviour in Shank2-mutant mice improved by restoring NMDA receptor function. Nature 2012, 486, 261-265. [CrossRef]

29. Schmeisser, M.J.; Ey, E.; Wegener, S.; Bockmann, J.; Stempel, A.V.; Kuebler, A.; Janssen, A.L.; Udvardi, P.T.; Shiban, E.; Spilker, C.; et al. Autistic-Like Behaviours and Hyperactivity in Mice Lacking Prosap1/Shank2. Nature 2012, 486, 256-260. [CrossRef]

30. Bozdagi, O.; Sakurai, T.; Papapetrou, D.; Wang, X.; Dickstein, D.L.; Takahashi, N.; Kajiwara, Y.; Yang, M.; Katz, A.M.; Scattoni, M.L.; et al. Haploinsufficiency of the autism-associated Shank3 gene leads to deficits in synaptic function, social interaction, and social communication. Mol. Autism 2010, 1, 15. [CrossRef]

31. Yang, M.; Bozdagi, O.; Scattoni, M.L.; Wohr, M.; Roullet, F.I.; Katz, A.M.; Abrams, D.N.; Kalikhman, D.; Simon, H.; Woldeyohannes, L.; et al. Reduced Excitatory Neurotransmission and Mild Autism-Relevant Phenotypes in Adolescent Shank3 Null Mutant Mice. J. Neurosci. 2012, 32, 6525-6541. [CrossRef] [PubMed]

32. Wang, X.; McCoy, P.A.; Rodriguiz, R.M.; Pan, Y.; Je, H.S.; Roberts, A.C.; Kim, C.J.; Berrios, J.; Colvin, J.S.; Bousquet-Moore, D.; et al. Synaptic Dysfunction and Abnormal Behaviors in Mice Lacking Major Isoforms of Shank3. Hum. Mol. Genet. 2011, 20, 3093-3108. [CrossRef] [PubMed]

33. Peça, J.; Feliciano, C.; Ting, J.T.; Wang, W.; Wells, M.F.; Venkatraman, T.N.; Lascola, C.D.; Fu, Z.; Feng, G. Shank3 mutant mice display autistic-like behaviours and striatal dysfunction. Nature 2011, 472, 437-442. [CrossRef] [PubMed] 
34. Kouser, M.; Speed, H.E.; Dewey, C.M.; Reimers, J.M.; Widman, A.J.; Gupta, N.; Liu, S.; Jaramillo, T.C.; Bangash, M.; Xiao, B.; et al. Loss of predominant Shank3 isoforms results in hippocampus-dependent impairments in behavior and synaptic transmission. J. Neurosci. 2013, 33, 18448-18468. [CrossRef]

35. Yates, J.R. Tuberous Sclerosis. Eur. J. Hum. Genet. 2006, 14, 1065-1073. [CrossRef] [PubMed]

36. Goorden, S.M.I.; Woerden, V.G.M.; Weerd, V.D.L.; Cheadle, J.P.; Elgersma, Y. Cognitive Deficits in Tsc1(+/-)Mice in the Absence of Cerebral Lesions and Seizures. Ann. Neuro. 2007, 62, 648-655. [CrossRef] [PubMed]

37. Tsai, P.T.; Hull, C.; Chu, Y.; Greene-Colozzi, E.; Sadowski, A.R.; Leech, J.M.; Steinberg, J.; Crawley, J.N.; Regehr, W.G.; Sahin, M. Autistic-like behaviour and cerebellar dysfunction in Purkinje cell Tsc1 mutant mice. Nature 2012, 488, 647-651. [CrossRef]

38. Reith, R.M.; McKenna, J.; Wu, H.; Hashmi, S.S.; Cho, S.-H.; Dash, P.K.; Gambello, M.J. Loss of Tsc2 in Purkinje cells is associated with autistic-like behavior in a mouse model of tuberous sclerosis complex. Neurobiol. Dis. 2013, 51, 93-103. [CrossRef] [PubMed]

39. Bonora, E.; The International Molecular Genetic Study of Autism Consortium (IMGSAC); Beyer, K.S.; A Lamb, J.; Parr, J.R.; Klauck, S.M.; Benner, A.; Paolucci, M.; Abbott, A.; Ragoussis, I.; et al. Analysis of reelin as a candidate gene for autism. Mol. Psychiatry 2003, 8, 885-892. [CrossRef]

40. Curran, T.; D'Arcangelo, G. Role of reelin in the control of brain development. Brain Res. Brain Res. Rev. 1998, 26, 285-294. [CrossRef]

41. Hadj-Sahraoui, N.; Frederic, F.; Delhaye-Bouchaud, N.; Mariani, J. Gender Effect on Purkinje Cell Loss in the Cerebellum of the Heterozygous Reeler Mouse. J. Neurogenet. 1996, 11, 45-58. [CrossRef]

42. Gharani, N.; Benayed, R.; Mancuso, V.; Brzustowicz, L.M.; Millonig, J.H. Association of the homeobox transcription factor, ENGRAILED 2, 3, with autism spectrum disorder. Mol. Psychiatry 2004, 9, 474-484. [CrossRef] [PubMed]

43. Fatemi, S.H.; Halt, A.R.; Realmuto, G.; Earle, J.; Kist, D.A.; Thuras, P.; Merz, A. Purkinje cell size is reduced in cerebellum of patients with autism. Cell. Mol. Neurobiol. 2002, 22, 171-175. [CrossRef] [PubMed]

44. Butler, M.G.; Dasouki, M.; Zhou, X.; Talebizadeh, Z.; Brown, M.; Takahashi, T.; Miles, J.; Wang, C.; Stratton, R.; Pilarski, R.; et al. Subset of individuals with autism spectrum disorders and extreme macrocephaly associated with germline PTEN tumour suppressor gene mutations. J. Med. Genet. 2005, 42, 318-321. [CrossRef] [PubMed]

45. Kwon, C.-H.; Luikart, B.W.; Powell, C.M.; Zhou, J.; Matheny, S.A.; Zhang, W.; Li, Y.; Baker, S.J.; Parada, L.F. Pten regulates neuronal arborization and social interaction in mice. Neuron 2006, 50, 377-388. [CrossRef] [PubMed]

46. Valluy, J.; Bicker, S.; Aksoy-Aksel, A.; Lackinger, M.; Sumer, S.; Fiore, R.; Wüst, T.; Seffer, D.; Metge, F.; Dieterich, C.; et al. A coding-independent function of an alternative Ube3a transcript during neuronal development. Nat. Neurosci. 2015, 18, 666-673. [CrossRef] [PubMed]

47. Kishino, T.; Lalande, M.; Wagstaff, J. UBE3A/E6-AP mutations cause Angelman syndrome. Nat. Genet. 1997, 15, 70-73. [CrossRef]

48. Nakatani, J.; Tamada, K.; Hatanaka, F.; Ise, S.; Ohta, H.; Inoue, K.; Tomonaga, S.; Watanabe, Y.; Chung, Y.J.; Banerjee, R.; et al. Abnormal behavior in a chromosome-engineered mouse model for human 15q11-13 duplication seen in autism. Cell 2009, 137, 1235-1246. [CrossRef]

49. Chadman, K.K. Animal models for autism in 2017 and the consequential implications to drug discovery. Expert Opin. Drug Discov. 2017, 12, 1187-1194. [CrossRef]

50. Bambini-Junior, V.; Rodrigues, L.; Behr, G.A.; Moreira, J.C.F.; Riesgo, R.; Gottfried, C. Animal model of autism induced by prenatal exposure to valproate: Behavioral changes and liver parameters. Brain Res. 2011, 1408, 8-16. [CrossRef]

51. Rodier, P.M.; Ingram, J.L.; Tisdale, B.; Nelson, S.; Romano, J. Embryological origin for autism: developmental anomalies of the cranial nerve motor nuclei. J. Comp. Neurol. 1996, 370, 247-261. [CrossRef]

52. Schneider, T.; Przewlocki, R. Behavioral Alterations in Rats Prenatally Exposed to Valproic Acid: Animal Model of Autism. Neuropsychopharmacology 2005, 30, 80-89. [CrossRef] [PubMed]

53. Wrenn, C.C. Social Transmission of Food Preference in Mice. Curr. Protoc. Neurosci. 2004, 28, 8.5G.1-8.5G.7. [PubMed]

54. Nicolini, C.; Fahnestock, M. The valproic acid-induced rodent model of autism. Exp. Neurol. 2018, 299, 217-227. [CrossRef] 
55. Edbauer, D.; Neilson, J.R.; Foster, K.A.; Wang, C.F.; Seeburg, D.P.; Batterton, M.N.; Tada, T.; Dolan, B.M.; Sharp, P.A.; Sheng., M. Regulation of Synaptic Structure and Function by Fmrp-Associated Micrornas Mir-125b and Mir-132. Neuron 2010, 65, 373-384. [CrossRef] [PubMed]

56. Zongaro, S.; Hukema, R.; D’Antoni, S.; Davidovic, L.; Barbry, P.; Catania, M.V.; Willemsen, R.; Mari, B.; Bardoni, B. The 3 Utr of Fmr1 Mrna Is a Target of Mir-101, Mir-129-5p and Mir-221: Implications for the Molecular Pathology of Fxtas at the Synapse. Hum. Mol. Genet. 2013, 22, 1971-1982. [CrossRef]

57. Urdinguio, R.G.; Fernández, A.F.; López-Nieva, P.; Rossi, S.; Huertas, D.; Kulis, M.; Liu, C.-G.; Croce, C.M.; Calin, G.A.; Esteller, M. Disrupted microRNA expression caused by Mecp2 loss in a mouse model of Rett syndrome. Epigenetics 2010, 5, 656-663. [CrossRef]

58. Taganov, K.D.; Boldin, M.P.; Chang, K.J.; Baltimore, D. Nf-Kappab-Dependent Induction of Microrna Mir-146, an Inhibitor Targeted to Signaling Proteins of Innate Immune Responses. Proc. Natl. Acad. Sci. USA 2006, 103, 12481-12486. [CrossRef]

59. Su, J.; Richter, K.; Zhang, C.; Gu, Q.; Li, L. Differential regulation of interleukin-1 receptor associated kinase 1 (IRAK1) splice variants. Mol. Immunol. 2007, 44, 900-905. [CrossRef]

60. Zhang, R.; Huang, M.; Cao, Z.J.; Qi, J.Y.; Qiu, Z.L.; Chiang, L.Y. Mecp2 Plays an Analgesic Role in Pain Transmission through Regulating Creb/Mir-132 Pathway. Mol. Pain 2015, 11. [CrossRef]

61. Lyu, J.-W.; Yuan, B.; Cheng, T.-L.; Qiu, Z.-L.; Zhou, W.-H. Reciprocal regulation of autism-related genes MeCP2 and PTEN via microRNAs. Sci. Rep. 2016, 6, 20392. [CrossRef] [PubMed]

62. Cheng, Y.; Wang, Z.M.; Tan, W.; Wang, X.; Li, Y.; Bai, B.; Li, Y.; Zhang, S.F.; Yan, H.L.; Chen, Z.L.; et al. Partial Loss of Psychiatric Risk Gene Mir137 in Mice Causes Repetitive Behavior and Impairs Sociability and Learning Via Increased Pde10a. Nat. Neurosci. 2018, 21, 1689-1703. [CrossRef] [PubMed]

63. Cortabitarte, A.D.S.; Berkel, S.; Cristian, F.-B.; Fischer, C.; Rappold, G.A. A direct regulatory link between microRNA-137 and SHANK2: implications for neuropsychiatric disorders. J. Neurodev. Disord. 2018, 10, 15. [CrossRef] [PubMed]

64. Vatsa, N.; Kumar, V.; Singh, B.K.; Kumar, S.S.; Sharma, A.; Jana, N.R. Down-Regulation of Mirna-708 Promotes Aberrant Calcium Signaling by Targeting Neuronatin in a Mouse Model of Angelman Syndrome. Front. Mol. Neurosci. 2019, 12, 35. [CrossRef]

65. Lackinger, M.; Sungur, A.O.; Daswani, R.; Soutschek, M.; Bicker, S.; Stemmler, L.; Wust, T.; Fiore, R.; Dieterich, C.; Schwarting, R.K.W.; et al. A Placental Mammal-Specific Microrna Cluster Acts as a Natural Brake for Sociability in Mice. Embo. Rep. 2019, 20. [CrossRef] [PubMed]

66. Hirsch, M.M.; Deckmann, I.; Fontes-Dutra, M.; Bauer-Negrini, G.; Nunes, G.D.-F.; Nunes, W.; Rabelo, B.; Riesgo, R.; Margis, R.; Bambini-Junior, V.; et al. Behavioral alterations in autism model induced by valproic acid and translational analysis of circulating microRNA. Food Chem. Toxicol. 2018, 115, 336-343. [CrossRef]

67. Siegel, G.; Obernosterer, G.; Fiore, R.; Oehmen, M.; Bicker, S.; Christensen, M.; Khudayberdiev, S.; Leuschner, P.F.; Busch, C.J.L.; Kane, C.; et al. A functional screen implicates microRNA-138-dependent regulation of the depalmitoylation enzyme APT1 in dendritic spine morphogenesis. Nature 2009, 11, 705-716. [CrossRef] [PubMed]

68. Dai, X.; Yin, Y.; Qin, L. Valproic Acid Exposure Decreases the Mrna Stability of Bcl-2 Via up-Regulating Mir-34a in the Cerebellum of Rat. Neurosci. Lett. 2017, 657, 159-165. [CrossRef]

69. Lin, X.; Guan, H.; Huang, Z.; Liu, J.; Li, H.; Wei, G.; Cao, X.; Li, Y. Downregulation of Bcl-2 Expression by miR-34a Mediates Palmitate-Induced Min6 Cells Apoptosis. J. Diabetes Res. 2014, 2014, 1-7. [CrossRef]

70. Loohuis, O.N.F.; Kole, K.; Glennon, J.C.; Karel, P.; Borg, G.V.D.; Gemert, Y.V.; Bosch, D.V.A.; Meinhardt, J.; Kos, A.; Shahabipour, F.; et al. Elevated Microrna-181c and Microrna-30d Levels in the Enlarged Amygdala of the Valproic Acid Rat Model of Autism. Neurobiol. Dis. 2015, 80, 42-53. [CrossRef]

71. Schneider, T.; Turczak, J.; Przewlocki, R. Environmental Enrichment Reverses Behavioral Alterations in Rats Prenatally Exposed to Valproic Acid: Issues for a Therapeutic Approach in Autism. Neuropsychopharmacology 2006, 31, 36-46. [CrossRef] [PubMed]

(C) 2019 by the authors. Licensee MDPI, Basel, Switzerland. This article is an open access article distributed under the terms and conditions of the Creative Commons Attribution (CC BY) license (http://creativecommons.org/licenses/by/4.0/). 